\title{
Penicilliumin B Protects against Cisplatin-Induced Renal Tubular Cell Apoptosis through Activation of AMPK-Induced Autophagy and Mitochondrial Biogenesis
}

\author{
Weiwei Shen ${ }^{a}$ Nan Jia $^{a}$ Jinhua Miao ${ }^{a}$ Shuangqin Chen ${ }^{a}$ Shan Zhou ${ }^{a}$ \\ Ping Meng ${ }^{a}$ Xuefeng Zhou ${ }^{b}$ Lan Tang ${ }^{c}$ Lili Zhou $^{\text {a,d }}$ \\ a State Key Laboratory of Organ Failure Research, National Clinical Research Center of Kidney Disease, Division of

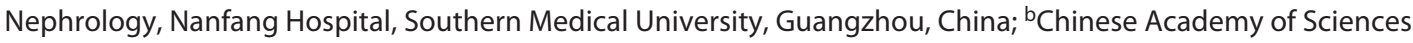 \\ (CAS) Key Laboratory of Tropical Marine Bio-resources and Ecology, Guangdong Key Laboratory of Marine Materia \\ Medica, South China Sea Institute of Oceanology, CAS, Guangzhou, China; 'Guangdong Provincial Key Laboratory of \\ New Drug Screening, School of Pharmaceutical Sciences, Southern Medical University, Guangzhou, China; ${ }^{\mathrm{d} B i o l a n d}$ \\ Laboratory (Guangzhou Regenerative Medicine and Health Guangdong Laboratory), Guangzhou, China
}

\section{Keywords}

Cisplatin · Apoptosis · Penicilliumin B · Autophagy ·

Adenosine $5^{\prime}$-monophosphate-activated protein kinase

\begin{abstract}
Introduction: Acute kidney injury (AKI) is at a high prevalence in hospitalized patients, especially in those receiving chemotherapy. Cisplatin is the most widely used chemotherapy drug; however, with its side effects that include nephrotoxicity, it also exhibits a risk of inducing AKI. Importantly, recent studies have shown that autophagy plays a protective role in cisplatin-induced AKI. However, therapeutic strategies and candidate drugs for inducing activation of autophagy remain limited. Methods: In the present study, we adopted a novel candidate drug from a deep sea-derived Penicillium strain, penicilliumin $B$, to testify its protective role in cisplatin-induced renal tubular cell injury. Results: Penicilliumin B exhibited protection against cisplatin-induced apoptosis in cultured renal tubular epithelial cells and in cisplatin-treated mice. Moreover, penicilliumin B maintained normal mitochondrial morphology and inhibited the pro-
\end{abstract}

karger@karger.com www.karger.com/kdd

Karger ${ }^{\prime \prime} \div$
(C) 2021 The Author(s)

Published by S. Karger AG, Basel

This is an Open Access article licensed under the Creative Commons Attribution-NonCommercial-4.0 International License (CC BY-NC) (http://www.karger.com/Services/OpenAccessLicense), applicable to the online version of the article only. Usage and distribution for commercial purposes requires written permission. duction of mitochondrial reactive oxygen species. Further studies demonstrated that penicilliumin $B$ enhanced autophagic flux, promoted the activation of multiple autophagy-related proteins, such as mTOR, Beclin-1, ATG5, PINK1, and LC3B, and induced the degradation of p62. Interestingly, we also found penicilliumin $B$ triggered phosphorylation of adenosine 5'-monophosphate-activated protein kinase (AMPK), which is an upstream inducer of nearly all autophagy pathways and also an activator of mitochondrial biogenesis. These results suggest that AMPK may represent an activated site of penicilliumin B. Consistently, compound $C$, an AMPK inhibitor, significantly blocked the protective effects of penicilliumin B on mitochondria and apoptotic inhibition. Conclusion: Taken together, our findings indicate that penicilliumin B represents a novel AMPK activator that may provide protection against renal tubular cell apoptosis through activation of AMPK-induced autophagy and mitochondrial biogenesis.

(c) 2021 The Author(s)

Published by S. Karger AG, Basel

Weiwei Shen and Nan Jia contributed equally to this work.
Correspondence to:

Lili Zhou, jinli730@smu.edu.cn 


\section{Introduction}

Acute kidney injury (AKI) is characterized by rapid deterioration in kidney function within a few days after the initial injury [1]. A large portion of patients with AKI undergo hospitalization, especially those receiving cardiac surgery and/or chemotherapy [2,3]. Patients with severe AKI, especially those in need of acute dialysis exhibit a high mortality rate [2]. Furthermore, a high proportion of surviving patients with AKI ultimately develop CKD [4]. However, to date, there is still no therapeutic drug for AKI.

The primary pathophysiology of AKI involves apoptosis or necrosis of renal tubular epithelial cells [5]. This pathogenesis can be induced by ischemia, sepsis, or nephrotoxins [6-8]. Among these factors, chemotherapeutic drugs induce renal tubular cell death when they are metabolized through the kidney [9]. Cisplatin is the most widely used chemotherapeutic drug for a variety of tumors [10, 11]; however, cisplatin also exhibits strong nephrotoxicity [12]. The mechanism of cisplatin-induced cell death may be related to inflammation, oxidative stress, endoplasmic-reticulum stress, and DNA damage [13-16]. Hence, considering these multifactorial deleterious effects of cisplatin, it is difficult for patients receiving chemotherapy to circumvent cisplatin-induced renal tubular cell injury, and there is still no effective therapeutics in the clinic to date.

Autophagy is an evolutionarily conserved self-defense process that helps to recycle nutrients and degrade waste within cells [17]. Autophagy plays a key role in maintaining physiological homeostasis but is also involved in the pathogenesis of several diseases [18, 19]. Many studies have shown that autophagy is highly involved in immunity [17], aging [20], and cancer progression [19] via regulation of inflammation [21], oxidative stress [22], and DNA damage/repair [23], each of which is a common characteristic feature in AKI. Interestingly, recent findings have shown that autophagy functions as a cytoprotective mechanism for cell survival in cisplatin-induced tubular cell apoptosis [24]. Even with the transient activation of autophagy, long-term exposure to cisplatin triggers a deficiency [25]. Therefore, candidate drugs that induce autophagy may help to mitigate cisplatin-induced apoptosis in renal tubular cells. However, currently available drugs with autophagy-enhancing activity are still limited.

Penicilliumin B is a novel sesquiterpene methylcyclopentenedione that was isolated from a sea-derived fungus, Penicillium sp. F00120, by our research group [26]. Our previous report has shown penicilliumin B suppress- es oxidative stress in cultured mesangial cells with low toxicity [26], suggesting that it exerts potential renoprotective effects. However, the therapeutic effects and underlying mechanisms of penicilliumin B on cisplatin-induced tubular injury remain unknown.

In the present study, we report that penicilliumin B protected against cisplatin-induced renal tubular epithelial cell injury in vitro and in vivo. The related mechanism involves enhanced activity of penicilliumin B on autophagy and mitochondrial biogenesis. The activated site of penicilliumin $B$ is highly related to adenosine 5'-monophosphate-activated protein kinase (AMPK), which is an upstream inducer of nearly all autophagic pathways and is also a strong activator of mitochondrial biogenesis. Taken together, our findings may help in guiding the development of novel intervention strategies for treating nephrotoxin-induced AKI.

\section{Materials and Methods}

\section{Animal Study}

Male C57BL/ 6 mice (8 weeks old) were purchased from the Experimental Animal Center of Southern Medical University. Mice were injected intraperitoneally with cisplatin at one dose of $30 \mathrm{mg} /$ $\mathrm{kg}$. Some mice were also subjected with intraperitoneal injections of penicilliumin $B$ on day 0 and day 1 at $2 \mathrm{mg} / \mathrm{kg}$. Mice were sacrificed at $72 \mathrm{~h}$ after injection of cisplatin. Meanwhile, 42 mice were used for animal survival analysis. All animal experiments were approved by the Animal Ethic Committee at Nanfang Hospital, Southern Medical University.

\section{Serum $\mathrm{Cr}$ and BUN Assay}

Serum Cr and BUN were assessed using an automatic chemistry analyzer (AU480; Beckman Coulter Inc; Kraemer Boulevard Brea, CA, USA).

\section{Cell Culture and Treatments}

Human proximal tubular epithelial cells (HKC-8) were provided by Dr. L. Racusen (Johns Hopkins University, Baltimore, MD, USA). Cells were cultured as described previously [27]. Some cells were treated with cisplatin (A5512; Sigma-Aldrich) in the absence or presence of penicilliumin B or compound C (S7840; Selleck). Some cells were also transfected with pCMV-GFP-LC3B plasmid to assess LC3B puncta.

\section{Terminal Deoxynucleotidyl Transferase dUTP Nick-End}

Labeling Assays

HKC-8 cells or kidney tissue were fixed with cold methanol:acetone (1:1) for $15 \mathrm{~min}$, and then incubated in TDT buffer containing TdT enzyme and FITC-conjugated dUTP according to the manufacturer's protocol (QIA39; Merck Millipore, Billerica, MA, USA). Apoptotic cells were counterstained with Hoechst 33258 (Merck Millipore, Billerica, MA, USA). Images were collected by fluorescent microscopy (Leica TCS SP2 AOBS, Leica Microsystems, Cambridge, UK). 


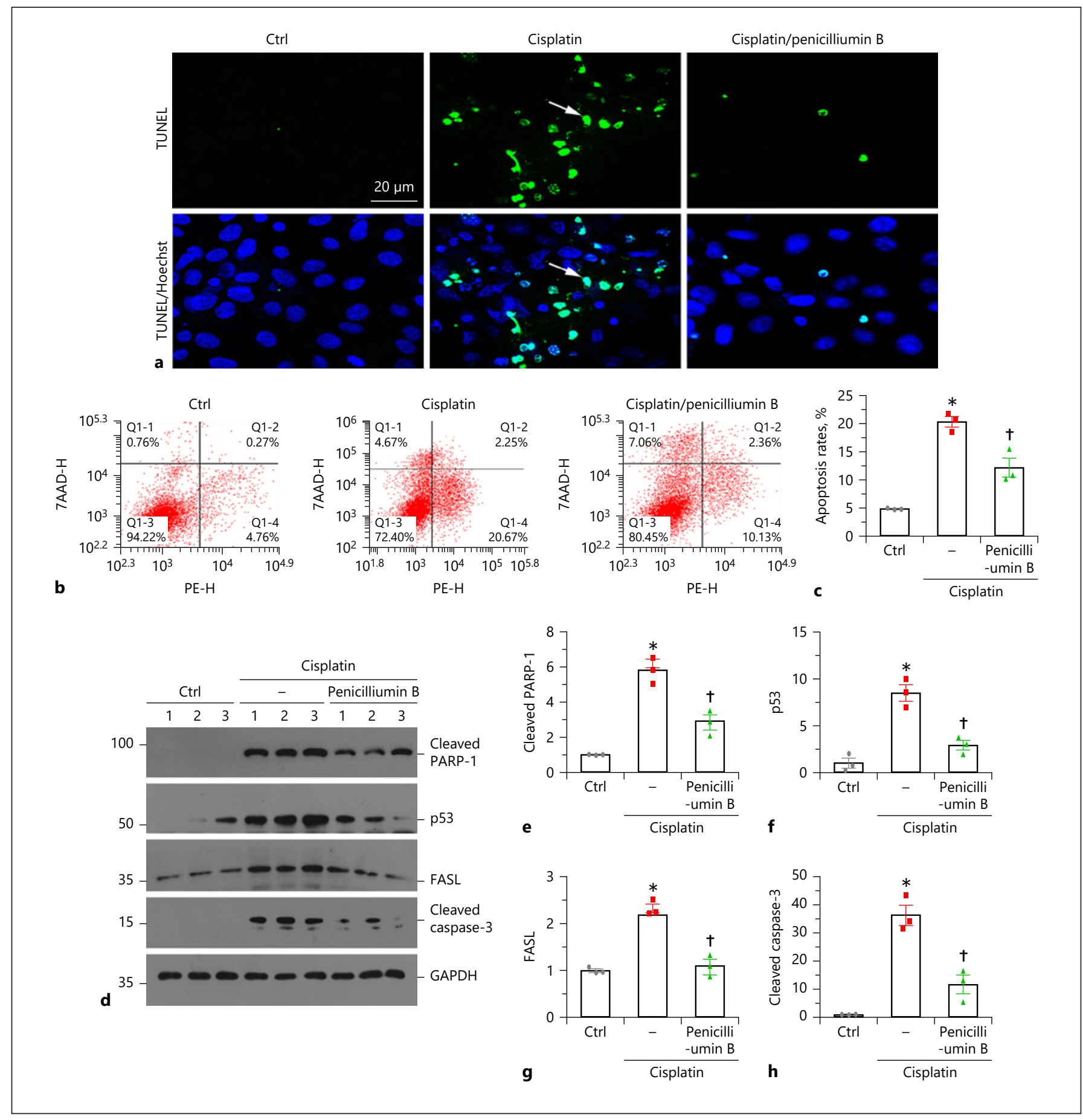

Fig. 1. Penicilliumin B protects tubular cells against cisplatin-induced apoptosis in vitro. a Representative micrographs show TUNEL-positive cells in different groups as indicated. HKC- 8 cells were stimulated by cisplatin $(20 \mu \mathrm{g} / \mathrm{mL})$ for $24 \mathrm{~h}$ with or without penicilliumin B $(0.5 \mu \mathrm{m})$. Arrows indicate TUNEL-positive apoptotic cells. b, c Representative fluorescence-activated cell sorter analyses show that penicilliumin $\mathrm{B}$ protected against cisplatin-induced apoptosis in HKC- 8 cells. HKC- 8 cells were stimulated by cisplatin $(20 \mu \mathrm{g} / \mathrm{mL})$ for $24 \mathrm{~h}$ with or without penicilliumin $\mathrm{B}(0.5 \mu \mathrm{m})$. The PE-A-positive cells were counted by flow cytometry. ${ }^{*} p<0.05$ versus Ctrl cells; ${ }^{\dagger} p<0.05$ versus cisplatin alone $(n=3)$. d-h Western blots show the expression of cleaved PARP-1, p53, FASL, and cleaved caspase-3 in the 3 groups, as indicated. HKC- 8 cells were stimulated by cisplatin $(20 \mu \mathrm{g} / \mathrm{mL})$ for $24 \mathrm{~h}$ with or without penicilliumin $\mathrm{B}(0.5 \mu \mathrm{m})$. Wholecell lysates were analyzed by Western blotting. ${ }^{*} p<0.05$ versus Ctrl cells; ${ }^{\dagger} p<0.05$ versus cisplatin alone $(n=3)$. TUNEL, terminal deoxynucleotidyl transferase dUTP nick-end labeling; PE-A, phycoerythrin-labeled annexin V; Ctrl, control. 

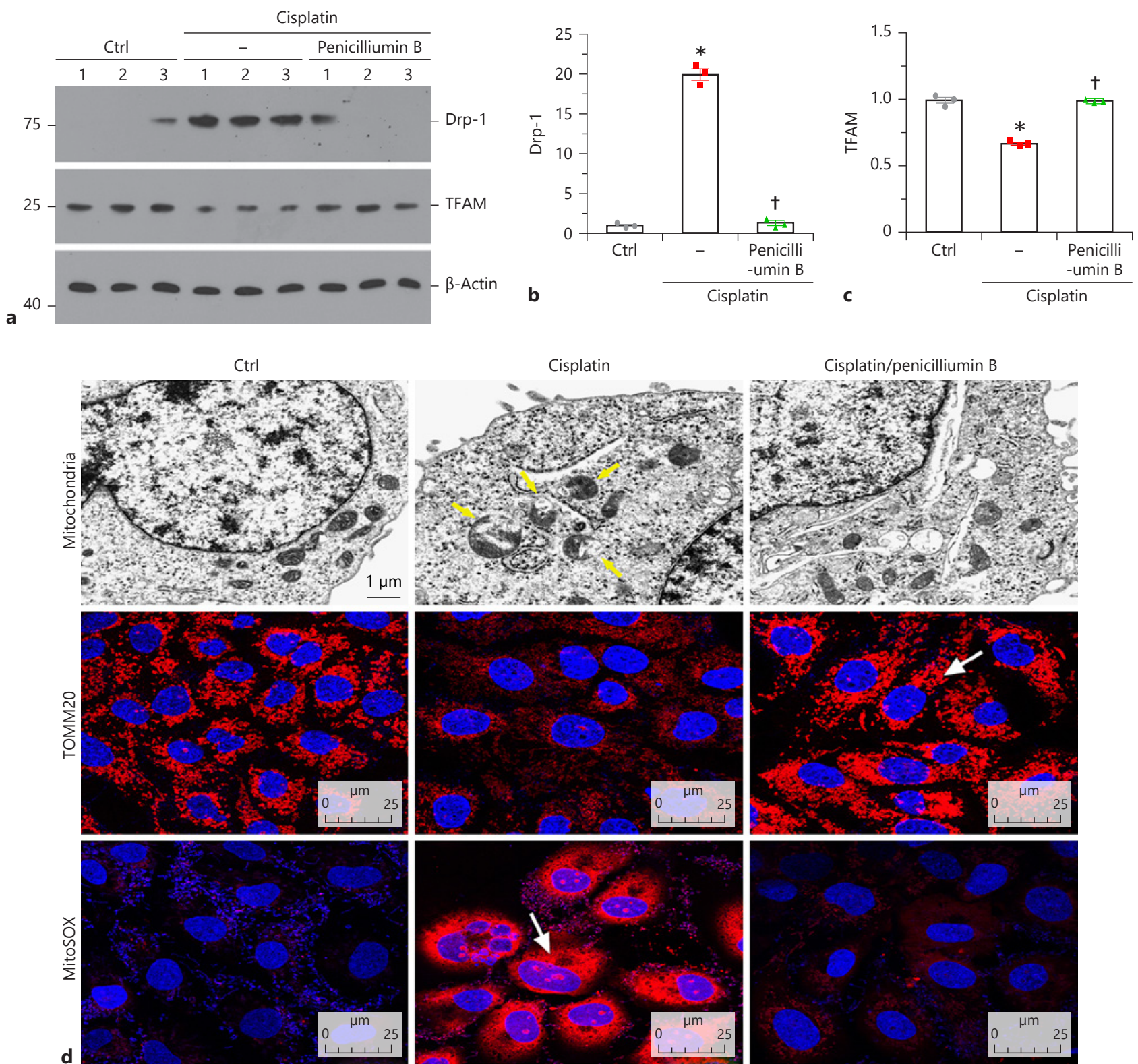

Fig. 2. Penicilliumin B preserves mitochondrial health in cisplatintreated tubular cells. a-c Representative Western blots show the expression of Drp1 and TFAM in the 3 groups, as indicated. HKC8 cells were stimulated by cisplatin $(20 \mu \mathrm{g} / \mathrm{mL})$ for $24 \mathrm{~h}$ with or without penicilliumin B $(0.5 \mu \mathrm{m}) .{ }^{*} p<0.05$ versus Ctrl cells; ${ }^{\dagger} p<$ 0.05 versus cisplatin alone $(n=3)$. d Representative TEM micrographs show penicilliumin $\mathrm{B}$ protects the normal structure of mitochondria in HKC-8 cells. Representative micrographs show
TOMM20 and MitoSOX staining. HKC- 8 cells were stimulated by cisplatin $(20 \mu \mathrm{g} / \mathrm{mL})$ for $24 \mathrm{~h}$ with or without penicilliumin B $(0.5$ $\mu \mathrm{m})$. DAPI ( $4^{\prime}, 6$-diamidino-2-phenylindole) was used to stain cellular nuclei (blue). For TEM analyses, arrows indicate abnormalshaped mitochondria. For TOMM20 and MitoSOX staining, arrows indicate positive staining. TFAM, transcription factor A, mitochondrial; TEM, transmission electron microscopy; Ctrl, control. 
Flow Cytometry Assay of Apoptosis

HKC-8 cells were treated in different groups as indicated. An Annexin V PE/7ADD kit (Multi Sciences biotech, Hangzhou, China) was used to analyze the apoptotic rate according to the manufacturer's instructions via flow cytometry (FACSCanto II, Becton Dickinson).

\section{Western Blotting}

Protein expression was analyzed by Western blot analyses as described previously [28]. Briefly, HKC-8 cells or kidney tissues were homogenized in lysis buffer and protein concentrations were measured using a Bradford assay, after which they were subjected to SDS-PAGE electrophoresis. After electrophoresis, the proteins were transferred to a PVDF membrane, blocked in blocking buffer ( $1 \%$ bovine serum albumin) for $1 \mathrm{~h}$, and were then incubated with primary antibodies overnight at $4^{\circ} \mathrm{C}$ and a secondary horseradish peroxidase-conjugated antibody for $1 \mathrm{~h}$ at room temperature. The antigen-antibody complexes were visualized using an ECL kit (Applygen, Beijing, China). The primary antibodies used were as follows: anti-PARP-1 (9542s, Cell Signaling Technology), anti-FasL (sc-19681,Santa Cruz Biotechnology), anti-p53 (sc-126; Santa Cruz Biotechnology), anti-cleaved caspase-3 (9661,Cell Signaling Technology), anti-Drp1 (ab184247, abcam), anti-TFAM (PB0413; Boster, Wuhan, China), anti-LC3B (ab48394, abcam), anti-mammalian target of rapamycin (mTOR) (ab32028, abcam), anti-pmTOR (ab109268, abcam), anti-Beclin-1 (ab62557, abcam), antiATG5 (BA3525-2, Boster), anti-PINK 1 (ab23707, abcam), antip62 (ab207305, abcam), anti-PGC-1a (ab54481, abcam), anti-p-AMPK (2535s, Cell Signaling Technology), anti-GAPDH (RM2002, Ray Antibody Biotech, Beijing, China), and anti- $\beta$-actin (RM2001, Ray Antibody Biotech, Beijing, China).

\section{Immunofluorescence and Immunohistochemistry Staining}

HKC-8 cells cultured on coverslips or frozen kidney sections ( 3 $\mu \mathrm{m})$ were fixed with $4 \%$ paraformaldehyde, permeabilized by $0.5 \%$ TritonX-100, and blocked with donkey serum. The slides were immunostained with primary antibodies as follows: anti-mTOR (ab32028, abcam), anti-p62 (ab207305, abcam), and antiTOMM20 (ab186735; Abcam). Paraffin kidney sections ( $3 \mu \mathrm{m})$ were performed immunohistochemistry staining using a routine protocol. The antibodies used were as follows: anti-cleaved caspase-3 (9661,Cell Signaling Technology) and anti-p-AMPK (2535s, Cell Signaling Technology).

Fig. 3. Penicilliumin B promotes autophagic flux in cultured tubular cells. a Representative electron microscopy (TEM) micrographs show that penicilliumin B increased autophagic vacuoles (yellow arrows). Representative micrographs at a larger magnification clearly present penicilliumin B could highly induce the formation of autophagolysosomes (red asterisks). HKC-8 cells were treated with penicilliumin B $(0.5 \mu \mathrm{m})$ for $24 \mathrm{~h}$. b Representative micrographs show penicilliumin B enhanced LC3BII formation. HKC- 8 cells were transiently transfected with GFP-LC3B plasmid for $24 \mathrm{~h}$ and were then stimulated with or without penicilliumin $\mathrm{B}$ $(0.5 \mu \mathrm{m})$ for $24 \mathrm{~h}$. The cells were observed by fluorescence microscopy. c-e Representative Western blots show the expression of LC3B and the ratio of LC3BII to LC3BI in the 3 groups, as indicated. HKC- 8 cells were treated with chloroquine $(10 \mu \mathrm{m})$ and co-
MitoSOX Staining

HKC-8 cells were cultured on coverslips and stained with MitoSOX (M36008, Thermo Fisher) according to the manufacturer's instructions.

\section{Transmission Electron Microscopy}

To assess mitochondrial morphology, HKC-8 cells and kidney tissues were collected and fixed in $1.25 \%$ glutaraldehyde $(0.1$ $\mathrm{mol} / \mathrm{L})$ in phosphate buffer. Ultrathin sections $(60 \mathrm{~nm})$ were prepared by a routine procedure and were examined under an electron microscope (JEOL JEM-1010, Tokyo, Japan).

\section{Detection of Autophagic Flux}

Cells were grown on coverslips and incubated with lentiviruses expressing RFP-GFP-LC3B (HANBIO, Shanghai, China), after which they were treated in different groups as indicated. The RFPGFP-LC3B sensor enables detection of neutral-pH LC3B-positive autophagosomes (green fluorescence) and acid-pH LC3B-positive autophagolysosomes (red fluorescence). Once an autophagosome fuses with a lysosome, the $\mathrm{pH}$ becomes acid and this leads to quenching of the GFP signal (whereas RFP is unaffected).

\section{Statistical Analyses}

All of the data are expressed as the mean \pm standard error of the mean. Statistical analysis of the data was carried out using SPSS 19.0 (SPSS Inc, Chicago, IL, USA). Comparisons between groups were made by $t$ tests when comparing 2 groups or via one-way ANOVA followed by Student-Newman-Keuls test or Dunnett's T3 procedure for comparison of more than 2 groups. A $p<0.05$ was considered to be statistically significant.

\section{Results}

\section{Penicilliumin B Protects Tubular Cells against \\ Cisplatin-Induced Apoptosis in vitro}

To identify the effects of penicilliumin B on apoptosis, we cultured human proximal tubular epithelial cells (HKC-8) and assayed apoptosis by terminal deoxynucleotidyl transferase dUTP nick-end labeling staining. As shown in Fig. 1a, apoptosis was induced in HKC-8 cells

treated with or without penicilliumin B $(0.5 \mu \mathrm{m})$ for $24 \mathrm{~h}$. Wholecell lysates were analyzed by Western blotting. ${ }^{\dagger} p<0.05$ versus chloroquine alone $(n=3)$. f, $\mathbf{g}$ Representative micrographs show that penicilliumin B promoted acidic-pH LC3B-positive autophagolysosomes (red fluorescence). HKC-8 cells were pretransfected with lentiviruses expressing RFP-GFP-LC3B for $24 \mathrm{~h}$ and were then stimulated by cisplatin $(20 \mu \mathrm{g} / \mathrm{mL})$ for $24 \mathrm{~h}$ with or without penicilliumin B $(0.5 \mu \mathrm{m})$. Natural-pH LC3B-positive autophagosomes (green fluorescence) and acidic-pH LC3B-positive autophagolysosomes (red fluorescence) were assessed. Quantitative data of autophagosomes (gold) and autophagolysosomes (red) are shown $(\mathbf{g}) .{ }^{*} p<0.05$ versus cisplatin alone (autophagosomes); ${ }^{\dagger} p<0.05$ versus cisplatin alone (autophagolysosomes) $(n=5)$. TEM, transmission electron microscopy.

(For figure see next page.) 


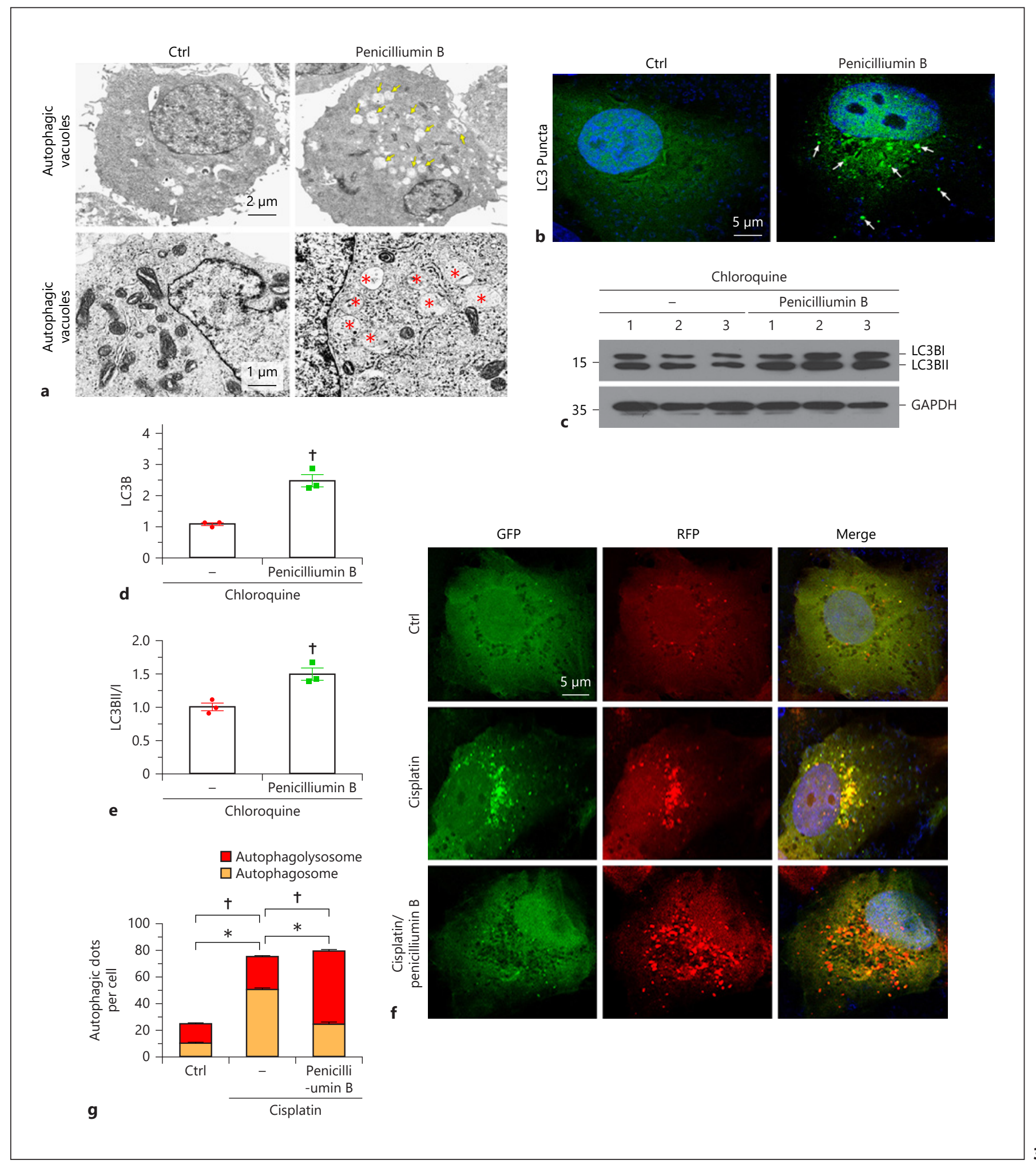


after treatment with cisplatin; however, cotreatment with penicilliumin B clearly inhibited cisplatin-induced apoptosis. We also quantified the ratio of apoptotic cells via Annexin V (AV)-labeling flow cytometry assay analysis. As shown in Fig. 1b, c, cisplatin-induced apoptosis was significantly blocked by cotreatment with penicilliumin $B$. We then assessed the expression levels of several apoptosis-related proteins such as FASL, P53, PARP-1, and cleaved caspase-3. As shown in Fig. 1d-h, the expression levels of all of these apoptosis-related proteins were increased via cisplatin; however, cotreatment with penicilliumin B significantly inhibited cisplatin-induced increases in the expression levels of these proteins.

\section{Penicilliumin B Preserves Mitochondrial Health in Cisplatin-Treated Tubular Cells}

We next assessed mitochondrial homeostasis in HKC8 cells. As shown in Fig. 2a, b, cisplatin stimulation promoted the upregulation of dynamin-related protein 1 (Drp1), a key mitochondrial fission protein [29]. However, cotreatment with penicilliumin B significantly inhibited this effect. We next analyzed transcription factor A, mitochondrial (TFAM), a master regulator governing mitochondrial biogenesis [28]. As shown in Fig. 2a, c, cisplatin stimulation decreased TFAM expression; however, cotreatment with penicilliumin B restored TFAM expression in the presence of cisplatin. Consistently, the ultrastructure of mitochondria, as assessed by transmission electron microscopy (TEM), further clarified the protective effects of penicilliumin B. As shown in Fig. 2d, cisplatin induced swollen mitochondria with disorganized and fragmented cristae in HKC-8 cells; however, cotreatment with penicilliumin B greatly improved the normal structure of mitochondria. Similar results were observed when TOMM20, the outer mitochondrial membrane protein translocase of outer mitochondrial membrane 20, was assessed by immunofluorescence, suggesting the protection of penicilliumin $\mathrm{B}$ on mito- chondrial mass (Fig. 2d). We also assessed mitochondrial reactive oxygen species (ROS) production via MitoSox staining. As shown in Fig. 2d, cotreatment with penicilliumin B inhibited cisplatin-induced mitochondrial ROS production.

\section{Penicilliumin B Promotes Autophagic Flux in} Cultured Tubular Cells

We next assessed the effects of penicilliumin B on autophagic flux. As shown in Fig. 3a, TEM images demonstrated that compared with that in normal cultured cells, penicilliumin $\mathrm{B}$ incubation induced an increase in autophagic vacuoles in HKC-8 cells. Furthermore, fluorescence of GFP-LC3B exhibited the formation of more LC3B puncta in penicilliumin B-treated cell (Fig. 3b). As chloroquine inhibits the fusion of autophagosome and lysosome, it is often used as a positive control for disrupted autophagic flux. We next assessed the effects of penicilliumin B on chloroquine-treated cells. As shown in Fig. 3c-e, cotreatment with penicilliumin B significantly enhanced the formation of LC3B and transformation of LC3BII from LCBI, which suggested an increase in autophagic flux [30]. To further assess autophagic flux, HKC-8 cells were infected with lentiviruses expressing a tandem RFP-GFP-LC3B fusion protein and were then treated with cisplatin or were cotreated with cisplatin/ penicilliumin B. The RFP-GFP-LC3B sensor enables LC3B-positive, neutral-pH autophagosomes as green fluorescence, whereas LC3B-positive acidic-pH autophagolysosomes exhibit red fluorescence. As shown in Fig. 3f, $\mathrm{g}$, cisplatin induced autophagosome accumulation without fusion of autophagosomes to lysosomes, as indicated by their golden color (overlap of red and green fluorescence). However, cotreatment with penicilliumin B triggered autophagic flux in HKC-8 cells, as shown by red puncta due to autophagosomes colocalizing with lysosomes (Fig. 3f, g).

p62 in the 3 groups, as indicated. HKC- 8 cells were stimulated by cisplatin $(20 \mu \mathrm{g} / \mathrm{mL})$ for $24 \mathrm{~h}$ with or without penicilliumin B $(0.5$ $\mu \mathrm{m}) .{ }^{*} p<0.05$ versus Ctrl cells; ${ }^{\dagger} p<0.05$ versus cisplatin alone $(n=3)$. $\mathbf{k}$ Representative micrographs show the expression of mTOR and p62 in the 3 groups. HKC- 8 cells were seeded on coverslips and stimulated by cisplatin $(20 \mu \mathrm{g} / \mathrm{mL})$ for $24 \mathrm{~h}$ with or without penicilliumin B $(0.5 \mu \mathrm{m})$. The cells were immunostained with an antibody against mTOR or p62. Arrows indicate positive staining. mTOR, mammalian target of rapamycin; PINK1, PTENinduced putative kinase protein 1; Ctrl, control.

(For figure see next page.) 


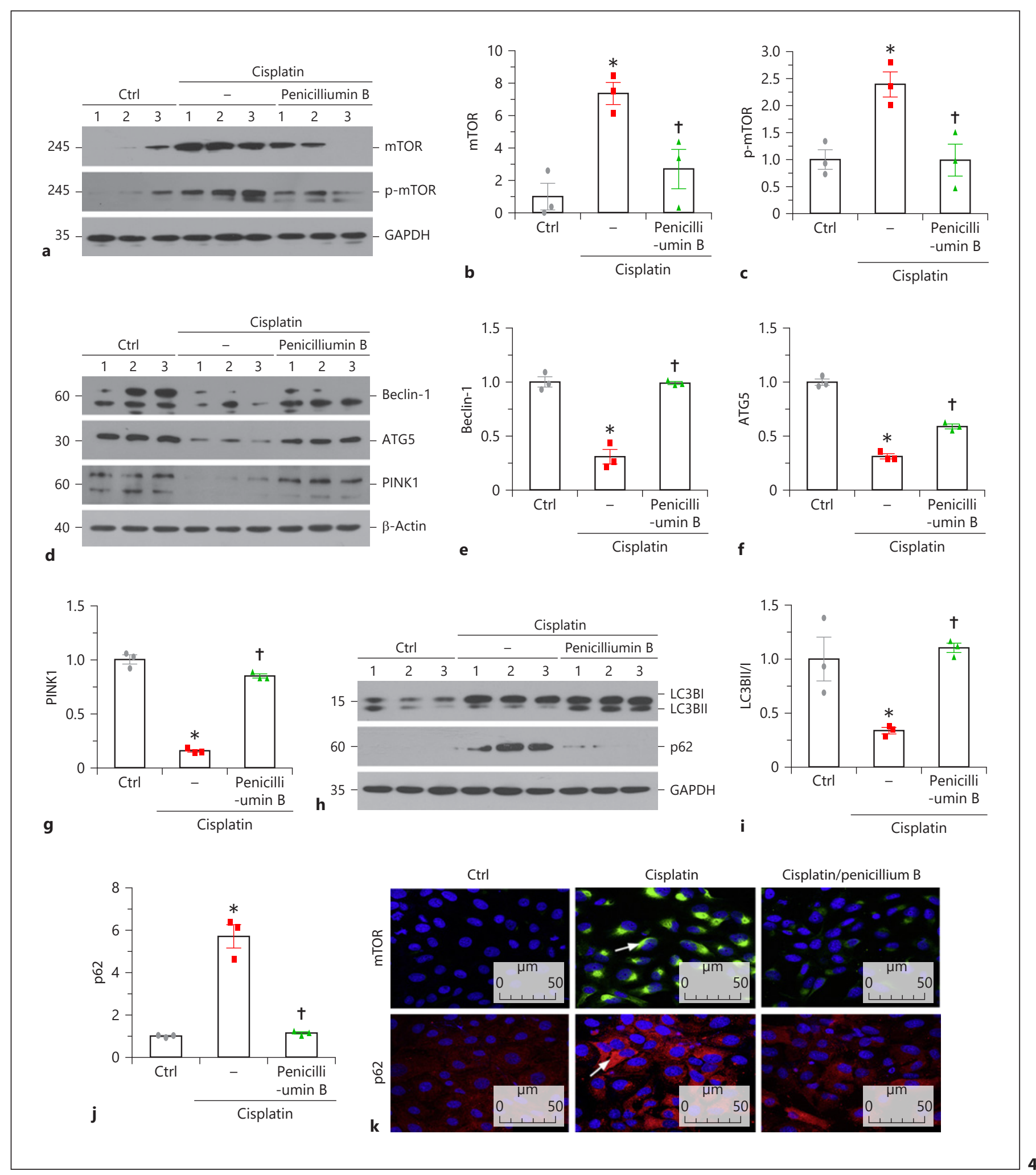


Penicilliumin B Increases Autophagic Pathways in Cisplatin-Treated Tubular Cells

To further clarify the underlying mechanisms of penicilliumin B in protection against cisplatin-induced tubular cell injury, we investigated autophagy-related (ATG) proteins. We first determined the expression of mTOR, an inhibitor of the uncoordinated 51-like kinase (ULK)1/ ULK2 complex that contributes to the initiation of autophagy [31]. As shown in Fig. 4a-c, cisplatin induced the upregulation of mTOR and its phosphorylated form. However, cotreatment with penicilliumin B greatly inhibited mTOR and its phosphorylation. Furthermore, penicilliumin B significantly reversed cisplatin-decreased Beclin-1 and ATG5, as shown in Fig. 4d-f. We next assessed the expression of PTEN-induced putative kinase protein 1 (PINK1), which is an initiator of mitophagy by anchoring to the outer membrane of depolarized mitochondria [32]. As shown in Fig. 4d, g, penicilliumin B effectively restored the expression of PINK1 in cisplatin-treated cells. In addition, the transformation of LC3BI to LC3BII was also greatly increased by cotreatment with penicilliumin $B$ in cisplatin-treated cells (Fig. 4h, i). Consistently, cisplatin induced increased expression of the autophagosome-selective substrate, p62 [33], but this expression was largely decreased by cotreatment with penicilliumin $\mathrm{B}$ (Fig. 4h, j). Similar results were observed when mTOR and p62 were assessed by immunofluorescence (Fig. 4k). These results suggest that penicilliumin $B$ is a potent inducer of autophagy by targeting multiple autophagic pathways.

\section{Penicilliumin B Protects against Renal Tubular Cell Apoptosis through Induction of AMPK}

Adenosine monophosphate kinase (AMPK) is an energy stress sensor that plays a central role in a variety of pathways [34], including autophagy and mitochondrial

Fig. 5. Penicilliumin B protects against renal tubular cell apoptosis through induction of AMPK. a-c Representative Western blots show the expression of p-AMPK and PGC-1 $\alpha$ in the 3 groups, as indicated. HKC- 8 cells were stimulated by cisplatin $(20 \mu \mathrm{g} / \mathrm{mL})$ for $24 \mathrm{~h}$ with or without penicilliumin B $(0.5 \mu \mathrm{m}) .{ }^{*} p<0.05$ versus Ctrl cells; ${ }^{\dagger} p<0.05$ versus cisplatin alone $(n=3)$. $\mathbf{d}$ Representative micrographs show the expression of TOMM20 in the 3 groups. HKC8 cells were seeded on coverslips and were pretreated with compound $\mathrm{C}(5 \mu \mathrm{m})$ for $1 \mathrm{~h}$ and were then stimulated by cisplatin $(20$ $\mu \mathrm{g} / \mathrm{mL})$ for $24 \mathrm{~h}$ with or without penicilliumin B $(0.5 \mu \mathrm{m})$. The cells were immunostained with an antibody against TOMM20. The arrow indicates positive staining. e-i Representative Western blots show the expression of p-AMPK, p-mTOR, PGC-1 $\alpha$, and cleaved caspase- 3 in the 3 groups, as indicated. HKC- 8 cells were pretreated with compound $\mathrm{C}(5 \mu \mathrm{m})$ for $1 \mathrm{~h}$, and then stimulated by cis- biogenesis $[35,36]$. Because we found that penicilliumin B regulated multiple autophagic pathways and protected mitochondrial homeostasis, we hypothesized penicilliumin B may be an activator of AMPK. Hence, we first tested the effects of penicilliumin B on p-AMPKa (Thr172), a phosphorylated and active form of AMPK [37]. As shown in Fig. 5a, b, treatment with penicilliumin B greatly ameliorated cisplatin-induced loss of p-AMPK. In addition, penicilliumin $B$ significantly restored the expression of peroxisome proliferator-activated receptor $\gamma$ coactivator-1a (PGC-1 $\alpha$ ), which is a downstream target activated by AMPK and a master regulator of mitochondrial biogenesis (Fig. 5a, c) [28, 38]. To further identify the role of penicilliumin B on AMPK activity, we treated HKC-8 cells with compound $\mathrm{C}$, a cell-permeable inhibitor of AMPK. As shown in Fig. 5d, cotreatment with compound C inhibited penicilliumin B-induced reversal of mitochondrial TOMM20 expression. In addition, in HKC-8 cells under stimulation from cisplatin, cotreatment with compound C significantly inhibited penicilliumin B-triggered restoration of p-AMPK and PGC-1 $\alpha$, as well as decrease in pmTOR and cleaved caspase-3 (Fig. 5e-i). Taken together, as summarized in Fig. 5j, we conclude that penicilliumin B serves as an activator of AMPK to further trigger autophagy. Notably, AMPK is a master regulator in modulating multiple pathways such as inducing Beclin-1 and ATG5, and inhibiting mTOR complex 1 (mTORC1) [39-41]. In addition, AMPK also activates PGC- $1 \alpha$ [38]. PGC- $1 \alpha$ is a key regulator of mitophagy through the Nrf2-induced PINK/Parkin pathway $[42,43]$ and plays a key role in mitochondrial biogenesis through induction of TFAM [28]. Through acting on these AMPK-regulated pathways, penicilliumin B may effectively trigger autophagic flux and induce mitochondrial biogenesis. All of these processes collectively contribute to mitochondrial health by reducing

platin $(20 \mu \mathrm{g} / \mathrm{mL})$ for $24 \mathrm{~h}$ with or without penicilliumin B $(0.5$ $\mu \mathrm{m})$. Whole-cell lysates were analyzed by Western blotting. ${ }^{*} p<$ 0.05 versus cisplatin alone; ${ }^{\dagger} p<0.05$ versus cisplatin plus penicilliumin $\mathrm{B}(n=3)$. $\mathbf{j}$ The schematic presentation depicts the potential mechanism by which penicilliumin B inhibits cell apoptosis in renal tubular cells. As an activator of AMPK, penicilliumin B may inhibit apoptosis by promoting autophagy and mitochondrial biogenesis. The underlying mechanisms are related to the activation of Beclin-1, ATG5, and PGC-1a, as well as inhibition of mTOR. These effects contribute to mitochondrial homeostasis and decreased mitochondrial ROS production and fragmentation, which collectively lead to reduced apoptosis. AMPK, adenosine 5'-monophosphate-activated protein kinase; Ctrl, control; PGC-1 $\alpha$, peroxisome proliferator-activated receptor $\gamma$ coactivator-1 $\alpha$; mTOR, mammalian target of rapamycin; ROS, reactive oxygen species.

(For figure see next page.) 
mitochondrial ROS production and inhibiting mitochondrial fragmentation, the effects of which subsequently inhibit cisplatin-induced apoptosis.

Penicilliumin B Protects against Kidney Injury and Apoptosis in Cisplatin-Treated Mice

To further identify the protective role of Penicilliumin $B$ in kidney injury, we performed animal study in cispla- tin-treated mice. The experimental design was shown in Fig. 6a. Administration of Penicilliumin B significantly increased the survival rate in cisplatin-treated mice (Fig. 6b) and efficiently decreased the levels of serum $\mathrm{Cr}$ (Fig. 6c) and BUN (Fig. 6d). Furthermore, Penicilliumin $\mathrm{B}$ also strongly inhibited the apoptosis-related proteins p53, FASL, and cleaved caspase-3 in cisplatin-treated mice (Fig. 6e-h). The similar results were also observed

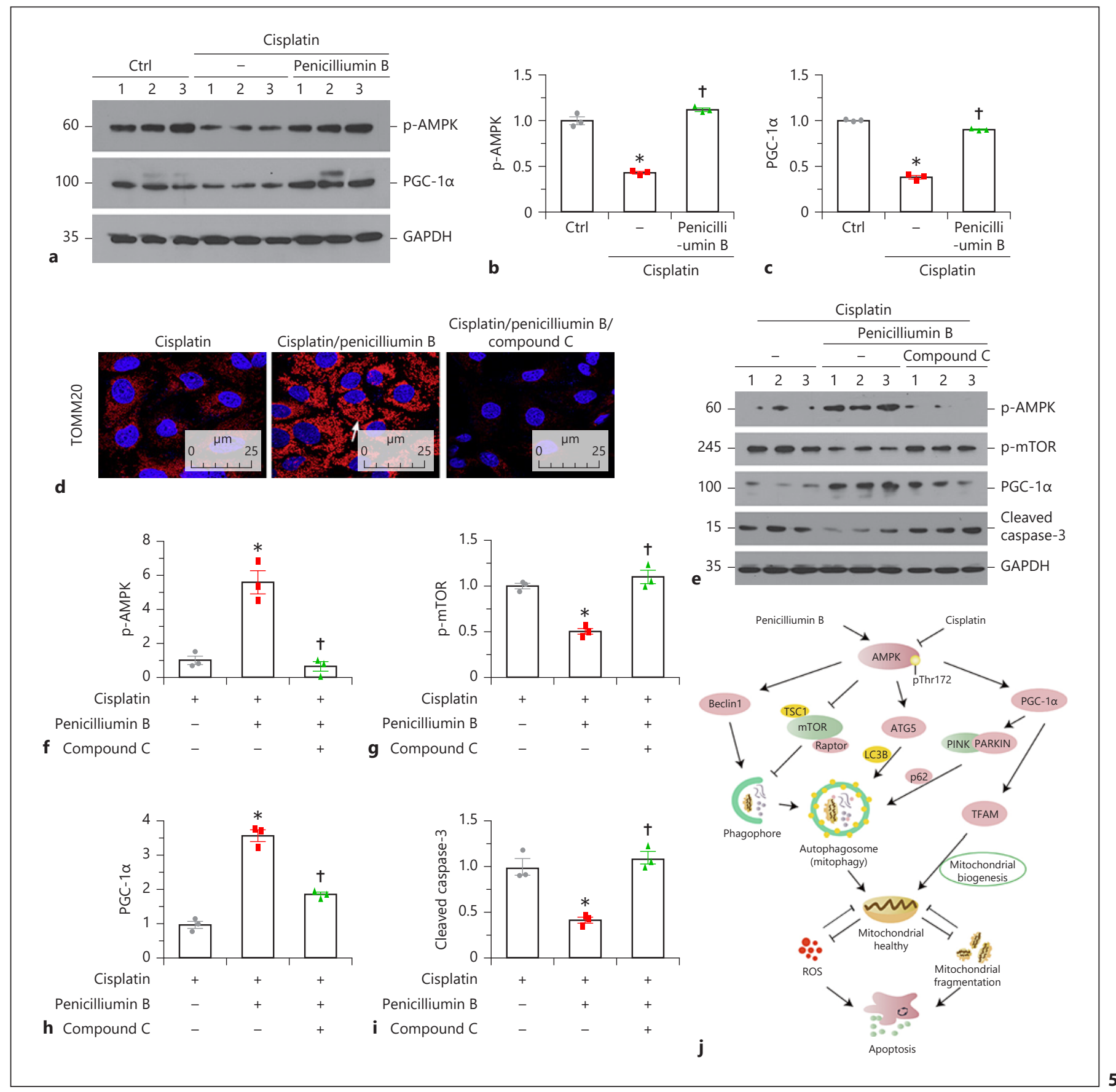

Penicilliumin B Inhibits Tubular Cell 


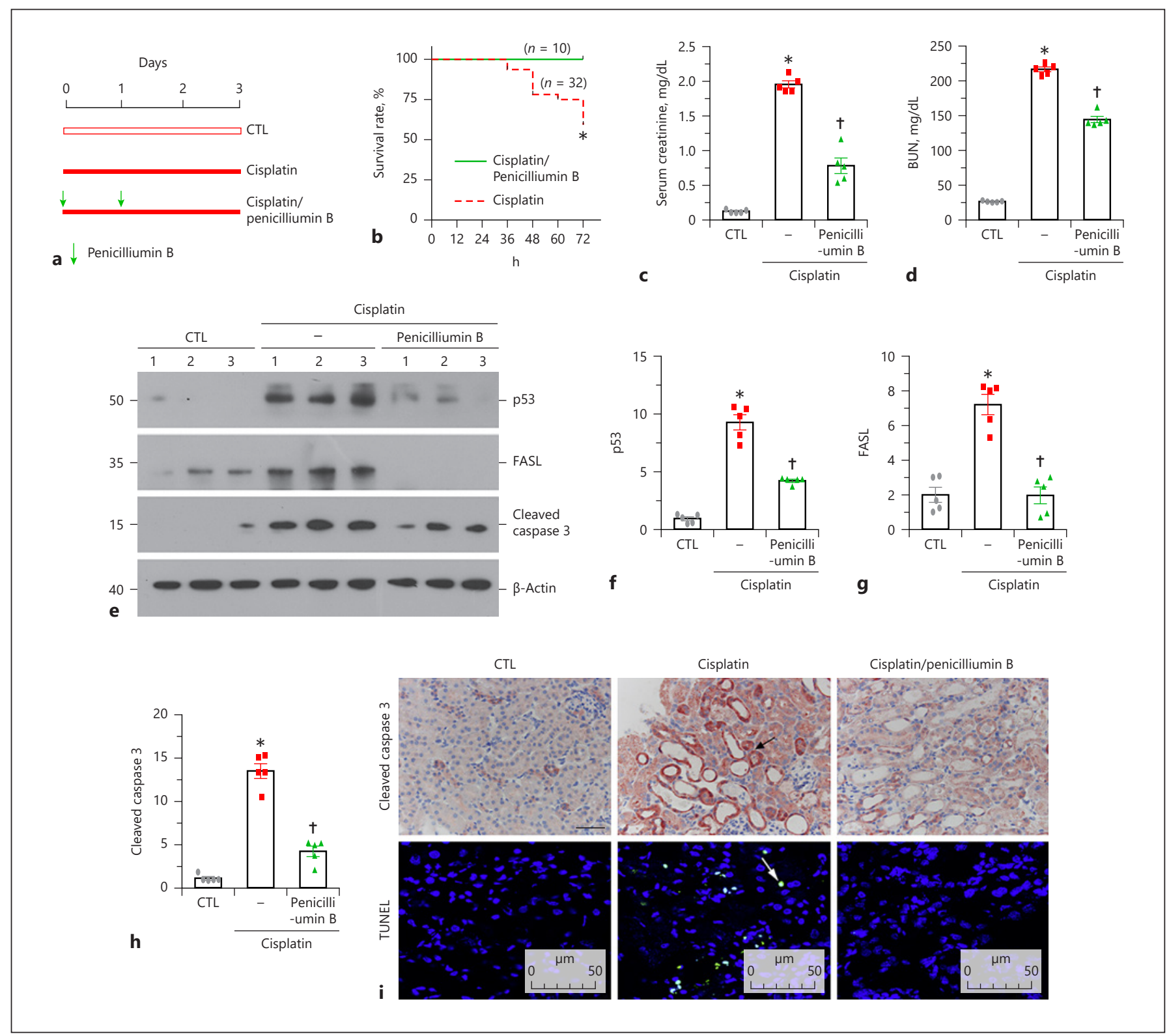

Fig. 6. Penicilliumin B protects against kidney injury and apoptosis in cisplatin-treated mice. a Experimental design. Green arrows indicate the injections of penicilliumin B. b Administration of penicilliumin B significantly decreases animal mortality rate in cisplatintreated mice. ${ }^{*} p<0.05$ versus cisplatin alone. c, $\mathbf{d}$ Graphic presentations show serum $\mathrm{Cr}$ and BUN levels in different groups. ${ }^{*} p<0.05$ versus Ctrl group; ${ }^{\dagger} p<0.05$ versus cisplatin alone $(n=5)$. e-h Representative Western blots and quantitative data show the expression

when cleaved caspase- 3 and positive apoptotic cells were tested by immunohistochemistry and terminal deoxynucleotidyl transferase dUTP nick-end labeling staining, respectively (Fig. 6i). of p53 (f), FASL ( $\mathbf{g})$, and cleaved caspase- $3(\mathbf{h}) .{ }^{*} p<0.05$ versus Ctrl group; ${ }^{\dagger} p<0.05$ versus cisplatin alone $(n=5)$. i Representative micrographs show the expression of cleaved caspase- 3 and TUNELpositive cells in the 3 groups. Arrows indicate positive staining. Paraffin sections were immunostained with an antibody against cleaved caspase-3. Frozen sections were performed with TUNEL staining. Arrows indicate positive staining. TUNEL, terminal deoxynucleotidyl transferase dUTP nick-end labeling; Ctrl, control.

Penicilliumin B Preserves Mitochondrial Biogenesis and Increases Autophagy in Cisplatin-Treated Mice through Promoting AMPK Activation

We next assessed the effects of Penicilliumin B on AMPK activity, mitochondrial biogenesis, and autophagy 
in cisplatin-treated mice. As shown in Fig. 7a, b, cotreatment with Penicilliumin B evidently increased the expression of $\mathrm{p}$-AMPK in cisplatin-treated mice. The similar result was also observed when p-AMPK was tested by immunostaining (Fig. 7c). Furthermore, cotreatment with Penicilliumin B triggered the restoration of PGC-1 $\alpha$ and TFAM (Fig. 7d-f), the 2 key factors of mitochondrial biogenesis. The similar results were also observed when TOMM20 was tested by immunofluorescence (Fig. 7g). We next analyzed the autophagic pathway. As shown in Fig. 7h-l, administration of Penicilliumin B in cisplatintreated mice significantly inhibited the expression of $\mathrm{p}$ mTOR (Fig. 7h, i) and upregulated the expression of ATG5 (Fig. 7h, j). Moreover, Penicilliumin B significantly increased the conversion of LC3I to LC3II (Fig. 7h, k) and decreased the accumulation of p62 (Fig. 7h, l), suggesting the inductive role of Penicilliumin B in autophagic flux. We next assessed the ultrastructure of mitchondria and autophagic vacuoles by TEM analysis. As shown in Fig. 7m, as compared with the normal round or rod-shaped and well-arranged mitochondria in control mice, treatment with cisplatin induced the disorganization of cristae arrangement and triggered numbers of swollen and broken mitochondria in renal proximal tubular epithelial cells (arrowheads). However, cotreatment with Penicilliumin B could largely preserve the normal morphology of mitochondria (Fig. $7 \mathrm{~m}$ ). We next assessed the autophagic vacuoles in 3 groups. As shown in Fig. $7 \mathrm{~m}$, although cisplatin triggered some autophagic vacuoles in tubular cells, cotreatment with Penicilliumin clearly promoted more autophagic vesicles of autophagosomes with surrounding double membrane (red arrows) and autophagolysosomes with single membrane (yellow arrows). These results further demonstrate Penicilliumin B protects against tubular injury through activation of AMPK and AMPK-induced mitochondrial biogenesis and autophagy.

\section{Discussion}

Autophagy plays a central role in maintaining cellular homeostasis [44]. In addition to playing a role in the recycling of nutrients, autophagy is essential for renal tubular cell survival under pathologic conditions, especially in term of apoptosis induced by nephrotoxins such as cisplatin [25]. Although cisplatin can induce transient upregulation of autophagy, long-term exposure of cisplatin damages autophagic functions in renal tubular cells [25]. Notably, the absence of autophagy accelerates the deterioration of renal functions.

Penicilliumin B Inhibits Tubular Cell

Apoptosis through AMPK
AMPK is a key regulator of energy metabolism by acting as a sensor of cellular energetic deficiencies [45]. AMPK can be activated by an increased ratio of intercellular AMP/ATP during nutrient deprivation, oxidative stress, and/or hypoxia [46-48]. The phosphorylation of AMPK leads to the activation of PGC-1 $\alpha$ [38], which results in mitochondrial biogenesis and ATP production by activating TFAM $[28,49]$. In addition, AMPK plays a key role in multiple pathways including autophagy [50]. Recent findings have shown that metformin, an activator of AMPK, exerts a protective role in renal tubular cells through autophagic induction [51], suggesting an inductive role of AMPK in autophagy. The underlying mechanisms may rely on the inhibitory effects of AMPK on mTORC1 activity. AMPK may also regulate Beclin-1, which determines the formation of phagophores. Furthermore, AMPK could induce LC3BII activity through its target, Sirt1 [52], to deacetylate ATG5 [53]. These findings suggest that AMPK activators serve as an autophagic inducers through multiple pathways. However, direct AMPK activators are still at early research stages and most are not clinically available [37]. In our present study, we found that penicilliumin B represents a novel AMPK activator that protects renal tubular cells through induction of autophagy.

There are several lines of evidence to support our findings. First, we assessed the effects of penicilliumin B on apoptosis in renal tubular cells. We found that penicilliumin $B$ protected against cellular apoptosis and regulated protein levels of PARP-1, p53, FASL, and cleaved caspase-3 (Fig. 1). Penicilliumin B also inhibited mitochondrial fission and ROS production and restored the normal structure of mitochondria in renal tubular cells (Fig. 2). We further identified that penicilliumin $\mathrm{B}$ induced autophagic flux and promoted the upregulation of a variety of ATG proteins (Fig. 3, 4). Furthermore, penicilliumin B triggered the phosphorylated activation of AMPK and its target, PGC-1a. Compound C, an AMPK inhibitor, significantly inhibited the protective effects of penicilliumin $\mathrm{B}$ on mitochondria, which further led to increased apoptosis (Fig. 5). We further testify the effects of penicilliumin B in cisplatin-treated mice. The results show penicilliumin B protects against kidney injury and tubular apoptosis and preserves mitochondrial biogenesis and increases autophagy, which is highly related with its inductive capability of AMPK activity (Fig. 6, 7). These results suggest that penicilliumin B acts as an AMPK activator to protect against renal tubular cell apoptosis through induction of autophagy.

Kidney Dis 2021;7:278-292

DOI: $10.1159 / 000514657$ 


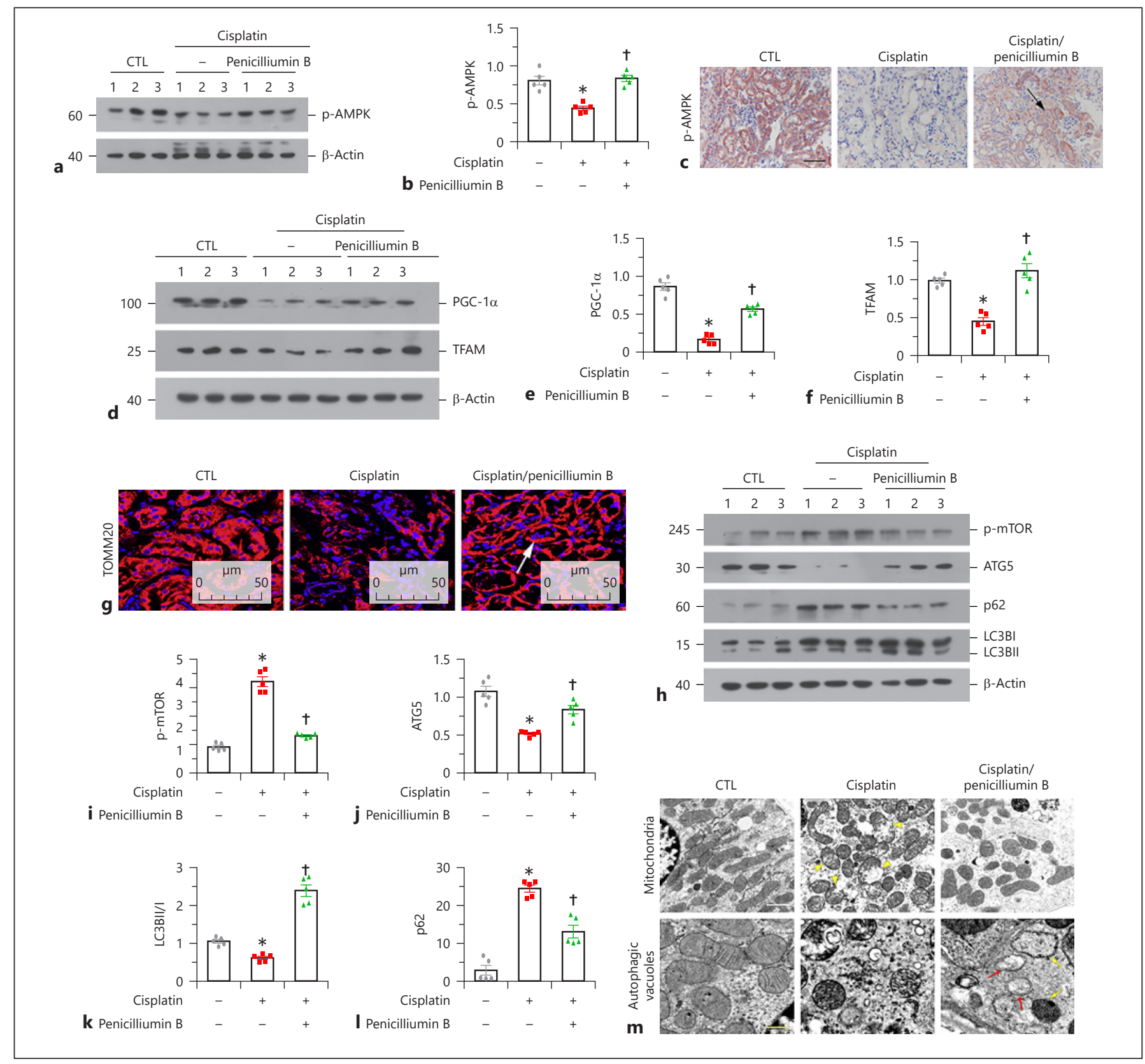

Fig. 7. Penicilliumin B preserves mitochondrial biogenesis and increases autophagy in cisplatin-treated mice through promoting AMPK activation. a, b Representative Western blots and quantitative data show the expression of $\mathrm{p}-\mathrm{AMPK}(\mathbf{b}) .{ }^{*} p<0.05$ versus Ctrl group; ${ }^{\dagger} p<0.05$ versus cisplatin alone $(n=5)$. c Representative micrographs show the expression of $\mathrm{p}$-AMPK in the 3 groups. Paraffin sections were immunostained with an antibody against $\mathrm{p}$ AMPK. Arrow indicates positive staining. d-f Representative Western blots and quantitative data show the expression of PGC$1 \alpha(\mathbf{e})$ and TFAM (f). ${ }^{*} p<0.05$ versus Ctrl group; ${ }^{\dagger} p<0.05$ versus cisplatin alone $(n=5)$. g Representative micrographs show the expression of TOMM20 in the 3 groups. Frozen sections were immunostained with an antibody against TOMM20. Arrow indicates positive staining. h-I Representative Western blots and quantita- tive data show the expression of p-mTOR (i), ATG5 (j), LC3II/I ratio (k), and p62 (I). ${ }^{*} p<0.05$ versus $C$ trl group; ${ }^{\dagger} p<0.05$ versus cisplatin alone $(n=5)$. $\mathbf{m}$ Ultrastructural analysis of mitochondrial morphology and autophagic vacuoles in proximal tubular cells. Ultrathin sections were observed using TEM. Yellow arrowheads indicate the broken mitochondria characterized by disorganization of cristae arrangement and swollen morphology. Red arrows indicate the autophagic vesicles of autophagosomes with surrounding double membrane. Yellow arrows indicate autophagolysosomes with single membrane (yellow arrows). AMPK, adenosine 5'-monophosphate-activated protein kinase; TEM, transmission electron microscopy; PGC-1 $\alpha$, peroxisome proliferatoractivated receptor $\gamma$ coactivator- $1 \alpha$; mTOR, mammalian target of rapamycin; Ctrl, control. 
Penicilliumin B is a novel sesquiterpene methylcyclopentenedione isolated from the deep sea-derived fungus, Penicillium sp. F00120. As a natural product, penicilliumin $B$ shows low toxicity and beneficial effects on oxidative stress and kidney fibrogenic action in culture mesangial cells, as previously reported by our group [25]. However, the underlying mechanisms have remained unclear. In the present study, we found that penicilliumin B induced autophagy in renal tubular cells. These effects were related to the activation of AMPK, which is a central regulator of energy homeostasis and a modulator of multiple important pathways including autophagy.

AMPK plays a key role in the development of obesity, cardiovascular disease, cancer, and kidney disease [37, 54]. The activation of AMPK greatly reduces proteinuria and improves kidney histology by suppressing mTOR $[55,56]$. However, pharmacological compounds that activate AMPK are mostly at early research stage and are not clinically available [37]. Only a few of these compounds are approved for clinical use in diabetes but not in kidney diseases [37]. In the present study, we found that penicilliumin B may represent a novel AMPK activator that strongly increases autophagic flux in renal tubular cells, which we found it protected against renal tubular cell apoptosis and maintained mitochondrial homeostasis. Although more studies are needed, our present findings may help to guide the development of novel therapeutic strategies for treating nephrotoxin-induced kidney injury.

\section{Statement of Ethics}

No human samples were used in this study. The animal experiments conform to internationally accepted standards and have been approved by the Animal Ethic Committee at Nanfang Hospital, Southern Medical University (No. NFYY-2020-84).

\section{Conflict of Interest Statement}

The authors have no conflicts of interest to declare.

\section{Funding Sources}

This work was supported by National Key R\&D Program of China (2020YFC2005000), National Natural Science Foundation of China Grant 82070707, 91949114, 81722011, 81521003, and the project of Innovation team of CKD with integrated traditional Chinese and Western Medicine (2019KCXTD014); Frontier Research Program of Guangzhou Regenerative Medicine and Health Guangdong Laboratory (2018GZR110105004); and Outstanding Scholar Program of Guangzhou Regenerative Medicine and Health Guangdong Laboratory (2018GZR110102004).

\section{Author Contributions}

Weiwei Shen, Nan Jia, Jinhua Miao, Shuangqin Chen, Shan Zhou, Ping Meng, Xuefeng Zhou, Lan Tang, and Lili Zhou conducted the experiments and prepared the materials involved in this study. Lili Zhou conceived this study. Lili Zhou participated in its design and coordination. Lili Zhou, Weiwei Shen, Nan Jia, and Jinhua Miao contributed to the analysis and interpretation of the data. Weiwei Shen and Lili Zhou drafted the manuscript. All authors read and approved the final manuscript.

\section{References}

1 Levey AS, James MT. Acute kidney injury. Ann Intern Med. 2017;167(9):ITC66-80.

2 Yang X, Chen C, Teng S, Fu X, Zha Y, Liu H, et al. Urinary matrix metalloproteinase-7 predicts severe AKI and poor outcomes after cardiac surgery. J Am Soc Nephrol. 2017;28(11): 3373-82.

3 Silver SA, Harel Z, McArthur E, Nash DM, Acedillo R, Kitchlu A, et al. Causes of death after a hospitalization with AKI. J Am Soc Nephrol. 2018;29(3):1001-10.

4 Hsu CY, Chinchilli VM, Coca S, Devarajan P, Ghahramani N, Go AS, et al. Post-acute kidney injury proteinuria and subsequent kidney disease progression: the assessment, serial evaluation, and subsequent sequelae in acute kidney injury (ASSESS-AKI) study. JAMA Intern Med. 2020;180(3):402-10.

5 Chen S, Fu H, Wu S, Zhu W, Liao J, Hong X, et al. Tenascin-C protects against acute kidney injury by recruiting Wnt ligands. Kidney Int. 2019;95(1):62-74.
6 Sun J, Zhang J, Tian J, Virzì GM, Digvijay K, Cueto L, et al. Mitochondria in sepsis-induced AKI. J Am Soc Nephrol. 2019;30(7):1151-61.

7 Nakazawa D, Kumar SV, Marschner J, Desai J, Holderied A, Rath L, et al. Histones and neutrophil extracellular traps enhance tubular necrosis and remote organ injury in ischemic AKI. J Am Soc Nephrol. 2017;28(6): 1753-68.

8 Liu J, Kumar S, Heinzel A, Gao M, Guo J, Alvarado GF, et al. Renoprotective and immunomodulatory effects of GDF15 following AKI invoked by ischemia-reperfusion injury. J Am Soc Nephrol. 2020;31(4):701-15.

9 Kong MJ, Bak SH, Han KH, Kim JI, Park JW, Park KM. Fragmentation of kidney epithelial cell primary cilia occurs by cisplatin and these cilia fragments are excreted into the urine. Redox Biol. 2019;20:38-45.

10 Yimit A, Adebali O, Sancar A, Jiang Y. Differential damage and repair of DNA-adducts induced by anti-cancer drug cisplatin across mouse organs. Nat Commun. 2019;10(1): 309.

11 Zhang R, Wang ZY, Li YH, Lu YH, Wang S, $\mathrm{Yu}$ WX, et al. Dynamic contrast-enhanced MRI to predict response to vinorelbine-cisplatin alone or with rh-endostatin in patients with non-small cell lung cancer and bone metastases: a randomised, double-blind, placebo-controlled trial. Lancet. 2016;388(Suppl 1):S95.

12 Gong J, Noel S, Hsu J, Bush EL, Arend LJ, Sadasivam $\mathrm{M}$, et al. TCR+CD4-CD8- (double negative) $\mathrm{T}$ cells protect from cisplatin-induced renal epithelial cell apoptosis and acute kidney injury. Am J Physiol Renal Physiol. 2020;318(6):F1500-12.

13 Xu M, Yu X, Meng X, Huang S, Zhang Y, Zhang A, et al. Inhibition of PDE4/PDE4B improves renal function and ameliorates inflammation in cisplatin-induced acute kidney injury. Am J Physiol Renal Physiol. 2020; 318(3):F576-88. 
14 Cao W, Yuan Y, Liu X, Li Q, An X, Huang Z, et al. Adenosine kinase inhibition protects against cisplatin-induced nephrotoxicity. Am J Physiol Renal Physiol. 2019;317(1):F10715.

15 Tan Z, Guo F, Huang Z, Xia Z, Liu J, Tao S, et al. Pharmacological and genetic inhibition of fatty acid-binding protein 4 alleviated cisplatin-induced acute kidney injury. J Cell Mol Med. 2019;23(9):6260-70.

16 Zhu S, Pabla N, Tang C, He L, Dong Z. DNA damage response in cisplatin-induced nephrotoxicity. Arch Toxicol. 2015;89(12):2197205.

17 Clarke AJ, Simon AK. Autophagy in the renewal, differentiation and homeostasis of immune cells. Nat Rev Immunol. 2019;19(3): $170-83$.

18 Devis-Jauregui L, Eritja N, Davis ML, MatiasGuiu X, Llobet-Navàs D. Autophagy in the physiological endometrium and cancer. Autophagy. 2020:1-19.

19 Bryant KL, Der CJ. Blocking autophagy to starve pancreatic cancer. Nat Rev Mol Cell Biol. 2019;20(5):265.

20 Lopez-Otín C, Kroemer G. Decelerating ageing and biological clocks by autophagy. Nat Rev Mol Cell Biol. 2019;20(7):385-6.

21 Bharath LP, Agrawal M, McCambridge G, Nicholas DA, Hasturk H, Liu J, et al. Metformin enhances autophagy and normalizes mitochondrial function to alleviate aging-associated inflammation. Cell Metab. 2020;32(1): 44-e6.

22 Yang Y, White E. Autophagy suppresses TRP53/p53 and oxidative stress to enable mammalian survival. Autophagy. 2020;16: $1355-7$.

23 Zheng K, He Z, Kitazato K, Wang Y. Selective autophagy regulates cell cycle in cancer therapy. Theranostics. 2019;9(1):104-25.

24 Liu J, Livingston MJ, Dong G, Tang C, Su Y, $\mathrm{Wu} \mathrm{G}$, et al. Histone deacetylase inhibitors protect against cisplatin-induced acute kidney injury by activating autophagy in proximal tubular cells. Cell Death Dis. 2018;9(3): 322.

25 Periyasamy-Thandavan S, Jiang M, Wei Q, Smith R, Yin XM, Dong Z. Autophagy is cytoprotective during cisplatin injury of renal proximal tubular cells. Kidney Int. 2008; 74(5):631-40.

26 Lin X, Wu Q, Yu Y, Liang Z, Liu Y, Zhou L, et al. Penicilliumin $B$, a novel sesquiterpene methylcyclopentenedione from a deep seaderived Penicillium strain with renoprotective activities. Sci Rep. 2017;7(1):10757.

27 Liu Y, Feng Q, Miao J, Wu Q, Zhou S, Shen $\mathrm{W}$, et al. C-X-C motif chemokine receptor 4 aggravates renal fibrosis through activating JAK/STAT/GSK3 $\beta / \beta$-catenin pathway. J Cell Mol Med. 2020;24(7):3837-55.

28 Miao J, Liu J, Niu J, Zhang Y, Shen W, Luo C, et al. Wnt/ $\beta$-catenin/RAS signaling mediates age-related renal fibrosis and is associated with mitochondrial dysfunction. Aging Cell. 2019;18(5):e13004.
29 Yuan Y, Zhang A, Qi J, Wang H, Liu X, Zhao $\mathrm{M}$, et al. p53/Drp1-dependent mitochondrial fission mediates aldosterone-induced podocyte injury and mitochondrial dysfunction. Am J Physiol Renal Physiol. 2018;314(5): F798-808.

30 Surolia R, Li FJ, Wang Z, Li H, Dsouza K, Thomas V, et al. Vimentin intermediate filament assembly regulates fibroblast invasion in fibrogenic lung injury. JCI Insight. 2019; 4(7):e123253.

31 Cinà DP, Onay T, Paltoo A, Li C, Maezawa Y, De Arteaga J, et al. Inhibition of MTOR disrupts autophagic flux in podocytes. J Am Soc Nephrol. 2012;23(3):412-20.

32 Yang YY, Gong DJ, Zhang JJ, Liu XH, Wang L. Diabetes aggravates renal ischemia-reperfusion injury by repressing mitochondrial function and PINK1/Parkin-mediated mitophagy. Am J Physiol Renal Physiol. 2019; 317(4):F852-64.

33 Aparicio R, Hansen M, Walker DW, Kumsta C. The selective autophagy receptor SQSTM1/ p62 improves lifespan and proteostasis in an evolutionarily conserved manner. Autophagy. 2020;16(4):772-4.

34 Garcia D, Shaw RJ. AMPK: mechanisms of cellular energy sensing and restoration of metabolic balance. Mol Cell. 2017;66(6):789-800.

35 Herzig S, Shaw RJ. AMPK: guardian of metabolism and mitochondrial homeostasis. Nat Rev Mol Cell Biol. 2018;19(2):121-35.

36 Gu X, Li Y, Chen K, Wang X, Wang Z, Lian $\mathrm{H}$, et al. Exosomes derived from umbilical cord mesenchymal stem cells alleviate viral myocarditis through activating AMPK/ mTOR-mediated autophagy flux pathway. J Cell Mol Med. 2020;24(13):7515-30.

37 Steinberg GR, Carling D. AMP-activated protein kinase: the current landscape for drug development. Nat Rev Drug Discov. 2019;18(7): $527-51$.

38 Jäger S, Handschin C, St-Pierre J, Spiegelman BM. AMP-activated protein kinase (AMPK) action in skeletal muscle via direct phosphorylation of PGC-1alpha. Proc Natl Acad Sci USA. 2007;104(29):12017-22.

39 Li MY, Zhu XL, Zhao BX, Shi L, Wang W, Hu $\mathrm{W}$, et al. Adrenomedullin alleviates the pyroptosis of Leydig cells by promoting autophagy via the ROS-AMPK-mTOR axis. Cell Death Dis. 2019;10(7):489.

40 Matsui Y, Takagi H, Qu X, Abdellatif M, Sakoda $\mathrm{H}$, Asano T, et al. Distinct roles of autophagy in the heart during ischemia and reperfusion: roles of AMP-activated protein kinase and Beclin 1 in mediating autophagy. Circ Res. 2007;100(6):914-22.

41 Wang S, Kandadi MR, Ren J. Double knockout of Akt2 and AMPK predisposes cardiac aging without affecting lifespan: Role of autophagy and mitophagy. Biochimica et Biophysica Acta (BBA) - Molecular Basis of Disease. 2019;1865(7):1865-75.

42 Xiao L, Xu X, Zhang F, Wang M, Xu Y, Tang D, et al. The mitochondria-targeted antioxidant MitoQ ameliorated tubular injury mediated by mitophagy in diabetic kidney disease via Nrf2/ PINK1. Redox Biol. 2017;11:297-311.

43 Vainshtein A, Tryon LD, Pauly M, Hood DA. Role of PGC-1a during acute exercise-induced autophagy and mitophagy in skeletal muscle. Am J Physiol, Cell Physiol. 2015; 308(9):C710-9.

44 Mizushima N, Komatsu M. Autophagy: renovation of cells and tissues. Cell. 2011;147(4): $728-41$.

45 Lin SC, Hardie DG. AMPK: Sensing Glucose as well as Cellular Energy Status. Cell Metab. 2018;27(2):299-313.

46 Dengler F. Activation of AMPK under Hypoxia: many roads leading to rome. Ijms. 2020;21(7):2428.

47 Wang C, Zhu L, Yuan W, Sun L, Xia Z, Zhang $Z$, et al. Diabetes aggravates myocardial ischaemia reperfusion injury via activating Nox2related programmed cell death in an AMPKdependent manner. J Cell Mol Med. 2020; 24(12):6670-9.

48 Sayama S, Song A, Brown BC, Couturier J, Cai $\mathrm{X}, \mathrm{Xu} \mathrm{P}$, et al. Maternal erythrocyte ENT1mediated AMPK activation counteracts placental hypoxia and supports fetal growth. JCI Insight. 2020;5(10):e130205.

49 Yin J, Nielsen M, Carcione T, Li S, Shi J. Apolipoprotein $\mathrm{E}$ regulates mitochondrial function through the PGC-1 1 -sirtuin 3 pathway. Aging. 2019;11(23):11148-56.

50 Gatica D, Klionsky DJ. New tricks of an old autophagy regulator: AMPK-dependent regulation of autophagy through CCNY (cyclin Y)-CDK16. Autophagy. 2020;16(6):973-4.

51 Li J, Gui Y, Ren J, Liu X, Feng Y, Zeng Z, et al. Metformin protects against cisplatin-induced tubular cell apoptosis and acute kidney injury via AMPKa-regulated autophagy induction. Sci Rep. 2016;6:23975.

52 Chyau C-C, Wang H-F, Zhang W-J, Chen C-C, Huang S-H, Chang C-C, et al. Antrodan alleviates high-fat and high-fructose diet-induced fatty liver disease in $\mathrm{C} 57 \mathrm{BL} / 6$ mice model via AMPK/Sirt1/SREBP-1c/PPAR $\gamma$ pathway. Int J Mol Sci. 2020;21(1):360.

$53 \mathrm{Hu} \mathrm{X,} \mathrm{Lu} \mathrm{Z,} \mathrm{Yu} \mathrm{S,} \mathrm{Reilly} \mathrm{J,} \mathrm{Liu} \mathrm{F,} \mathrm{Jia} \mathrm{D,} \mathrm{et} \mathrm{al.}$ CERKL regulates autophagy via the NAD-dependent deacetylase SIRT1. Autophagy. 2019; 15(3):453-65.

54 Lu YH, Chang YP, Li T, Han F, Li CJ, Li XY, et al. Empagliflozin attenuates hyperuricemia by upregulation of ABCG2 via AMPK/AKT/ CREB signaling pathway in type 2 diabetic mice. Int J Biol Sci. 2020;16(3):529-42.

55 Gwon DH, Hwang TW, Ro JY, Kang YJ, Jeong JY, Kim DK, et al. High endogenous accumulation of $\omega-3$ polyunsaturated fatty acids protect against ischemia-reperfusion renal injury through AMPK-mediated autophagy in fat-1 mice. Int J Mol Sci. 2017;18(10):2081.

56 Satriano J, Sharma K, Blantz RC, Deng A. Induction of AMPK activity corrects early pathophysiological alterations in the subtotal nephrectomy model of chronic kidney disease. Am J Physiol Renal Physiol. 2013;305(5): F727-33. 